\title{
Correlations of Perceived Deficits Questionnaire of Multiple Sclerosis Quality of Life Inventory with Beck Depression Inventory and neuropsychological tests
}

\author{
Jesus Lovera, MD, MSPH; ${ }^{1-2^{*}}$ Bridget Bagert, MD, MPH; ${ }^{3}$ Kyle H. Smoot, MD; ${ }^{2}$ Katherine Wild, PhD; ${ }^{2}$ Rachel \\ Frank, BA; ${ }^{2}$ Kristin Bogardus, BS; ${ }^{2}$ Barry S. Oken, MD; ${ }^{2}$ Ruth H. Whitham, MD; ${ }^{1-2}$ Dennis N. Bourdette, MD ${ }^{1-2}$ \\ ${ }^{1}$ Multiple Sclerosis Center of Excellence West, Portland Department of Veterans Affairs (VA) Medical Center, \\ Portland, OR; ${ }^{2}$ Department of Neurology, Oregon Health \& Science University School of Medicine, Portland, OR; \\ ${ }^{3}$ Department of Environmental Health, Harvard School of Public Health, Cambridge, MA
}

\begin{abstract}
The Perceived Deficits Questionnaire (PDQ) is a part of the Multiple Sclerosis (MS) Quality of Life Inventory that assesses self-perceived cognitive difficulties. We used baseline data from 49 MS subjects participating in a clinical trial to evaluate the correlation of the PDQ with two measures of cognitive impairment, the Paced Auditory Serial Addition Test (PASAT) and the California Verbal Learning Test, 2nd edition (CVLT-II), total score, and one measure of depression, the Beck Depression Inventory-Amended (BDI-IA). The PDQ correlated significantly $(r=0.42$; 95\% confidence interval [CI], 0.15 to $0.62 ; p=0.003$ ) with the BDI-IA scores but not with either the PASAT ( $r=-0.22$; 95\% CI, -0.48 to $0.06 ; p=0.2$ ) or the CVLT-II total ( $r=-0.17 ; 95 \% \mathrm{CI},-0.43$ to $0.12 ; p=$ $0.25)$. A subset of 38 of these subjects who scored worse than 0.5 standard deviation below the mean on the PASAT or CVLT-II received a more extensive neuropsychological battery of tests. No significant correlations were found between any of these tests and the PDQ. These results suggest that self-perceived cognitive dysfunction relates more to depression than to objective cognitive dysfunction.
\end{abstract}

Key words: Beck Depression Inventory, California Verbal Learning Test, cognition disorders, depression, multiple sclerosis, neuropsychological tests, Paced Auditory Serial Addition Test, Perceived Deficits Questionnaire, quality of life, questionnaires.

\section{INTRODUCTION}

Patients with multiple sclerosis (MS) can experience deterioration in their quality of life (QOL) in physical, cognitive, and emotional domains. Instruments that measure the impact of MS in health-related QOL have been developed to capture the impact of the disease on other domains besides physical disability. The prevalence of cognitive dysfunction in people with MS has been

Abbreviations: BDI-IA = Beck Depression Inventory-Amended; $\mathrm{CI}=$ confidence interval; COWAT = Controlled Oral Word Association Test; CVLT-II = California Verbal Learning Test, 2nd edition; MS = multiple sclerosis; MSQLI = MS Quality of Life Inventory; PASAT $=$ Paced Auditory Serial Addition Test; PDQ = Perceived Deficits Questionnaire; QOL = quality of life; $\mathrm{SD}=$ standard deviation; SDMT = Symbol Digit Modalities Test; Stroop = Stroop color-word test; UFOV = useful field of view; VA = Department of Veterans Affairs.

*Address all correspondence to Jesus Lovera, MD, MSPH; Department of Veterans Affairs (VA) Multiple Sclerosis Center of Excellence West, Portland VA Medical Center, 3710 SW U.S. Veterans Hospital Road, Portland, OR 97239-2999; 503-220-8262, ext. 54770; fax: 503-721-1422. Email: loveraj@ohsu.edu

DOI: 10.1682/JRRD.2004.09.0118 
estimated to be between 45 and 65 percent [1-2]. Cognitive impairment in people with MS is associated with decreased employment and social interactions [3]. Instruments for measuring QOL in MS should include useful scales that measure the impact of cognitive impairment. The MS QOL Inventory (MSQLI) is a modular MSspecific health-related QOL instrument consisting of a widely used generic measure, the 36-item short form Medical Outcomes Study Health Status Questionnaire that is supplemented by nine symptom-specific measures [4]. The Perceived Deficits Questionnaire (PDQ) is a part of the MSQLI and measures self-reported symptoms of psychological impairment. The MSQLI has been shown to have internal reliability and construct validity in a large sample of North American subjects [4]. To understand the contributions of objective impairment in standard neuropsychological tests and depression to self-perception of cognitive deficits, we used baseline data of 49 subjects to evaluate the correlation of the PDQ with the Beck Depression Inventory-Amended (BDI-IA) and with two neuropsychological tests, the Paced Auditory Serial Addition Test (PASAT) and the total score from the California Verbal Learning Test, 2nd edition (CVLT-II). We selected the PASAT and the CVLT-II total score because these two tests measure sustained attention and memory, respectively. These domains are frequently impaired in people with MS [1-2]. The PASAT has also been routinely used in clinical trials in MS and is part of the MS Functional Composite. Both tests are easy to administer and have good population norms. In a subgroup of 38 subjects with below-average performance on the CVLT-II total score or the PASAT, we also determined the correlation of the PDQ with a more extensive battery of tests that included the Controlled Oral Word Association Test (COWAT), the Symbol Digit Modalities Test (SDMT), an adapted version of the useful field of view (UFOV), and the University of Victoria version of the Stroop color-word test (Stroop). We chose the SDMT and the Stroop as additional measures of attention and the COWAT as a measure of verbal fluency because performance on all three tests is frequently impaired in MS [1]. We chose the UFOV, although not extensively studied in MS, because it provides a measure of cognitive processing speed that is independent of a timed motor response. Research has shown that subjects with MS who are cognitively impaired perform worse on this test than healthy controls [5-6].

\section{METHODS}

\section{Subjects}

To conduct the analysis, we used baseline data from MS subjects who volunteered for a double-blind placebocontrolled trial of Ginkgo biloba for the treatment of cognitive impairment. The Oregon Health \& Science University Institutional Review Board approved the study before initiation. All subjects who participated in the study signed an informed consent. For entry of participants into the parent Ginkgo biloba clinical trial, inclusion criteria were-

1. Diagnosis of MS according to McDonald et al. criteria [7].

2. Ages 18 to 60 .

3. Subjective cognitive impairment.

4. English as primary language.

Exclusion criteria were-

1. Score greater than 19 (moderate depression on the BDI-IA) [8].

2. Requirement of a permanent caregiver.

3. Significant medical diseases such as insulin-dependent illnesses, uncontrolled hypertension, uncontrolled hypothyroidism, liver or kidney failure, significant lung disease, alcoholism or other drug abuse, a known bleeding diathesis, symptoms or signs of congestive heart failure, angina, or significant valvular disease.

4. Visual impairment worse than 20/50 (binocular).

5. Pregnancy.

6. Significant exacerbation in the prior 30 days.

Subjects who fulfilled the inclusion and exclusion criteria received the PASAT and CVLT-II. Subjects who scored worse than 0.5 standard deviation (SD) below the reported means for either test qualified for the treatment phase of the parent clinical trial, completed the PDQ, and underwent more detailed neuropsychological testing at the same visit [1,9]. Subjects who scored better than 0.5 SD below the mean on both tests did not enter the parent clinical trial and received a PDQ in the mail that they completed and returned. The primary study analysis includes all subjects (49), and a subgroup analysis includes subjects (38) who participated in the parent Gingko biloba trial and received the more detailed neuropsychological battery. 


\section{Instruments}

All subjects included in this report received the PASAT, CVLT-II, BDI-IA, and PDQ. Only those who scored worse than 0.5 SD below the mean on the PASAT or CVLT-II received the rest of the neuropsychological battery that included the COWAT [10], SDMT [11], an adapted version of the UFOV [12], and the Stroop [13].

The PASAT [11] is a frequently used cognitive test that measures working memory and sustained attention. Subjects are presented with a number every 3 seconds and instructed to add the latest number with the one immediately before. Approximately 25 percent of subjects with MS are impaired on this test [1].

The CVLT-II is a test of memory and learning. Subjects listen to a list of 16 items belonging to four categories presented at one item a second and are then requested to repeat as many as they can remember. This procedure is repeated in five trials and the score for each trial is recorded. The total score (CVLT-II total) is the sum of the five trials. An interference trial with a different list of 16 items is then performed and the subjects are asked to recall this new list. This test is followed by subjects being asked to recall the items in the initial list (CVLT-II short delay free) and then again after they are provided with the categories as cues (CVLT-II short delay cued). After 20 minutes performing a distracter task, the subjects are asked to recall the initial items spontaneously (CVLT-II long delay free) and again after being provided with the categories as cues (CVLT-II long delay cued). In similar tests of learning and recent memory, 31 percent of subjects with MS are impaired [1].

The BDI-IA is a self-report screening instrument for depression. It consists of 21 items that measure the severity of depression in adults [8].

The PDQ consists of 20 items that address cognitive difficulties. Subjects rate their responses on a 5-point scale ranging from 1 (never) to 5 (almost always). The scale is made up of the following questions:

1. Lose your train of thought when speaking?

2. Have difficulty remembering the names of people, even the ones you have met several times?

3. Forgot what you came into the room for?

4. Have trouble getting things organized?

5. Have trouble concentrating on what people are saying during a conversation?

6. Forget if you have already done something?

7. Miss appointments and meetings you had scheduled?

8. Have difficulties planning what to do in the day?
9. Have trouble concentrating on things like watching a television program or reading a book?

10. Forget what you did the night before?

11. Forget the date unless you looked it up?

12. Have trouble getting started, even if you had a lot of things to do?

13. Find your minding drifting?

14. Forgot what you talked about after a telephone conversation?

15. Forgot to do things like turn off the stove or turn on your alarm clock?

16. Feel like your mind went totally blank?

17. Have trouble holding phone numbers in your head, even for a few seconds?

18. Forget what you did last weekend?

19. Forget to take your medication?

20. Have trouble making decisions?

The COWAT is a measure of phonemic verbal fluency and consists of three word-naming trials [10]. The examiner asks subjects to say as many words as they can think of in 1 minute. Every word in the list is to begin with the same given letter. Each of the three trials uses a different letter for a total of three letters.

The SDMT assesses information processing speed and visual tracking [11]. Subjects are presented with rows of blank squares, each of which is associated with a symbol. Above these rows is a key that matches the symbols with given numbers. The task of the subject is to fill in the blank squares with the corresponding numbers using the key. The subjects give their answers verbally. The total number of correct responses in 90 seconds is the score.

The UFOV is a computer-based test of visual information processing, divided attention, and selective attention that quantifies the visual field area over which a patient can process rapidly presented visual information [12]. The test consists of a brief presentation of the stimulus image. The stimulus image is either a car or a van in the center of the screen and a car in one of eight possible peripheral locations. The subject is asked to determine whether the car or the van was presented and to identify in which of eight possible locations the peripheral car was located. The test measures cognitive processing speed independently of timed motor response. We modified the original version to determine a precise perceptual threshold. The testing determines the minimum stimulus duration necessary for the subject to respond correctly 75 percent of the time. In addition to the threshold determination, the 
number of errors made at durations above threshold is calculated. The number of errors above threshold divided by the number of trials results in the percentage error rate above threshold. This error rate reflects lapses in attention since the subject is known to be able to respond accurately at these stimuli durations.

The Stroop is a measure of concentration, attention, and mental flexibility [13]. The examiner shows subjects a card with a list of color names. Each color is printed in an ink of a color that does not match the name of the color; for example, "blue" is printed in green ink. The color names and the colors of the ink can be blue, green, yellow, or red. The order of the color names in the list and the color of the ink in which they are printed are random. The task is to name the color of each word, and the outcome is the time the subject takes to complete the list.

\section{Statistical Analyses}

We performed statistical analyses using proc CORR (correlation procedure) in SAS ${ }^{\circledR}$ version 8.01 (SAS Institute Inc, Cary, North Carolina). We screened individual variables for normality using normal probability plots, histograms, and the Kolmogorov-Smirnov goodness-of-fit test. The PASAT showed moderate negative skewness that was corrected with a negative square root transformation. The Stroop and the UFOV also showed substantial positive skewness that was corrected with a log transformation. Scatter plots did not show significant deviations from assumptions of linear correlation analysis. Observations with extreme values or high influence in the diagnostics were checked for accuracy. Pearson's correlation coefficients $(r)$ between the PDQ and BDI-IA and the PASAT and CVLT-II total score were computed for all 49 subjects. We also analyzed a subset of 38 of these subjects who scored worse than 0.5 SD below the mean on the PASAT or CVLT-II. Pearson's correlation coefficients between the PDQ, BDI-IA, COWAT, SDMT, UFOV, and Stroop were computed for this subset. We calculated confidence intervals (CIs) for the correlation coefficients using Fisher's transformation and normal approximation [14]. A p-value of 0.05 was considered significant for this part of the analysis.

As an exploratory analysis, correlations between the individual items of the CVLT-II and PASAT were computed. Bonferroni's correction for multiple comparisons was used for this part of the analysis, because the conservative criteria $p$-values $<0.0008$ were considered significant.

\section{RESULTS}

\section{Study Profile}

The study profile is presented in Figure 1. Fifty-seven subjects fulfilled the inclusion and exclusion criteria and received the PASAT and CVLT-II. Of these subjects, 39 had scores on the CVLT-II or PASAT between 0.5 and 2.5 SD below the mean and qualified for the treatment phase of the clinical trial. These 39 subjects received the complete neuropsychological battery. Of these 39 subjects, 38 completed the PDQ form. Four subjects in this group did not complete one of the 20 questions in the PDQ. Two of them omitted question eight, one omitted question six, and one omitted question four. The missing values were replaced by the mean response for the whole

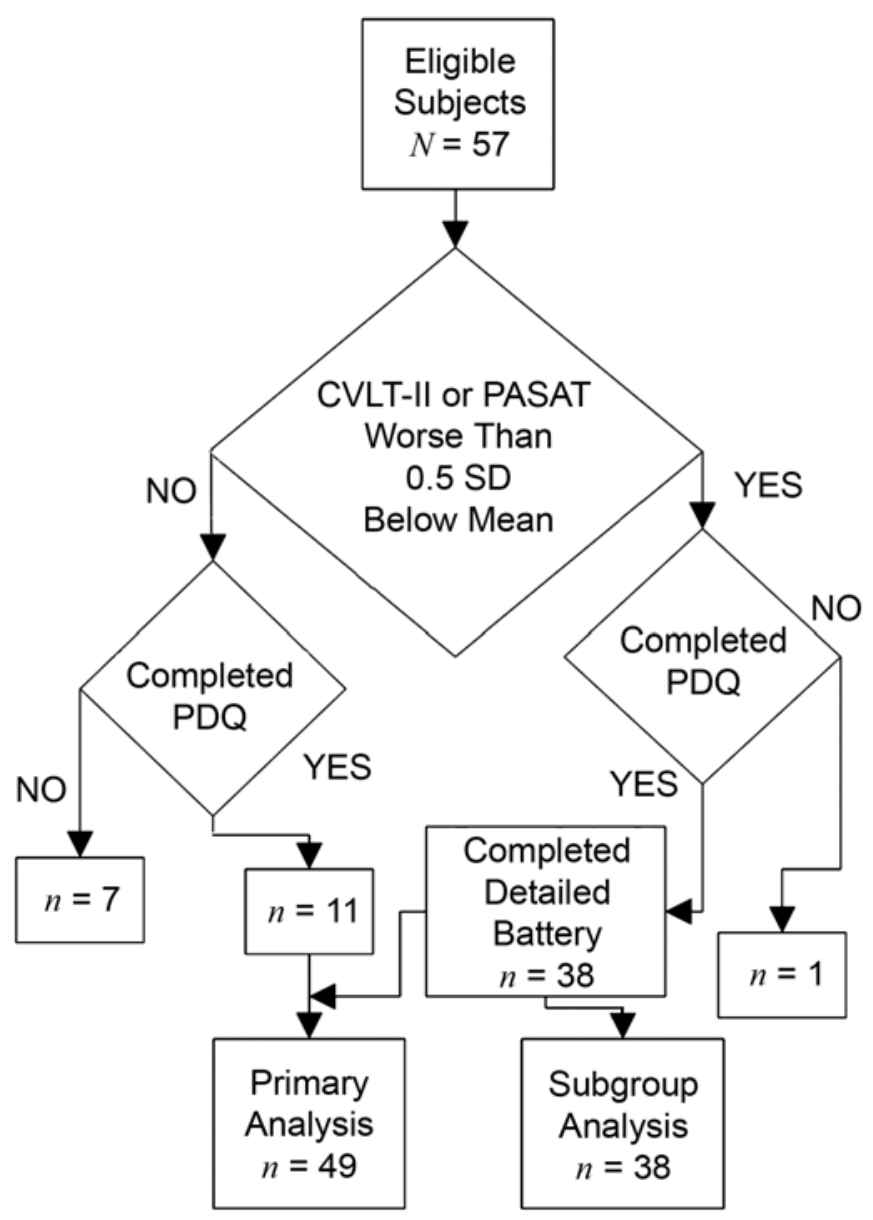

Figure 1.

Study profile of multiple sclerosis subjects who completed Perceived Deficits Questionnaire (PDQ), Paced Auditory Serial Addition Test (PASAT), and California Verbal Learning Test, 2nd edition (CVLT-II), cognitive impairment measures. SD = standard deviation. 
sample. The analysis was repeated excluding these four subjects (not shown). Eighteen subjects did not have significant impairment in either the CVLT-II or PASAT. Of these subjects, 11 completed the PDQ. These 11 subjects answered all of the questions.

\section{Demographics}

Table 1 presents the demographics for the 49 subjects who completed the PDQ, BDI, PASAT, and CVLT-II total score. The sex distribution was similar to that expected from a population sample of MS subjects with more females than males. Table 2 shows the mean and SD scores for the PASAT, CVLT-II, BDI-IA, and PDQ for all subjects. Figure 2 shows the distribution of the $z$ scores for the PASAT and CVLT-II total score on all subjects. The mean \pm SD scores for the PASAT were similar to those seen in a large MS population study [1]. Of the subjects, 73 percent

Table 1.

Demographic characteristics for all subjects $(n=49)$, mean age $49.3 \pm$ 7.6 standard deviation.

\begin{tabular}{lc}
\hline \multicolumn{1}{c}{ Variable } & Number (\%) \\
\hline Sex & $12(24)$ \\
Male & $37(76)$ \\
Female & \\
Race/Ethnicity & $46(94)$ \\
Caucasian & $1(2.0)$ \\
African American & $0(0)$ \\
Asian or Pacific Islander & $0(0)$ \\
Native American & $0(0)$ \\
Hispanic & $2(4.1)$ \\
Other & \\
Type of Multiple Sclerosis & $32(65)$ \\
Relapsing-Remitting & $15(31)$ \\
Secondary Progressive & $2(4.1)$ \\
Primary Progressive &
\end{tabular}

Table 2.

Mean \pm standard deviation (SD) scores for neuropsychological tests and Perceived Deficits Questionnaire (PDQ) for all subjects $(n=49)$.

\begin{tabular}{lc}
\hline Variable & Mean \pm SD \\
\hline PASAT & $39.0 \pm 12.7$ \\
CVLT-II Total & $45.4 \pm 12.0$ \\
BDI-IA & $6.9 \pm 5.0$ \\
PDQ & $54.6 \pm 12.7$ \\
\hline
\end{tabular}

PASAT = Paced Auditory Serial Addition Test, BDI-IA = Beck Depression Inventory-Amended, CVLT-II = California Verbal Learning Test, 2nd edition. had no depression (BDI-IA score $<9$ ) and 27 percent had mild depression according to the BDI-IA (BDI-IA score 10-18).

Table 3 shows mean \pm SD score for the rest of the neuropsychological tests on the 38 subjects who received the complete neuropsychological battery, and Figure 3 shows the distribution of the $z$ scores. The mean Expanded Disability Status Scale of this subset was 3.6 \pm 1.4 SD.

\section{Correlations Between PASAT, CVLT-II, and BDI-IA with PDQ of All Subjects}

The PASAT and the CVLT-II total score were significantly, though modestly, correlated ( $r=-0.31$; 95\% CI, 0.54 to $-0.02 ; p=0.03)$. The BDI-IA did not correlate with either the PASAT ( $r=0.19$; 95\% CI, -0.10 to $0.45 ; p=$ 0.19 ) or CVLT-II total score ( $r=-0.27 ; 95 \%$ CI, -0.51 to $0.02 ; p=0.06$ ). The PDQ did not correlate with either the PASAT ( $r=0.18$; 95\% CI, -0.11 to $0.44 ; p=0.20$ ) or CVLT-II total score $(r=-0.17$; $95 \%$ CI, -0.43 to $0.12 ; p=$ $0.25)$. The PDQ did correlate significantly with the BDI-IA ( $r=0.42$; $95 \% \mathrm{CI}, 0.15$ to $0.62 ; p=0.003$ ). Table 4 shows the correlation matrix along with the respective $p$-values.

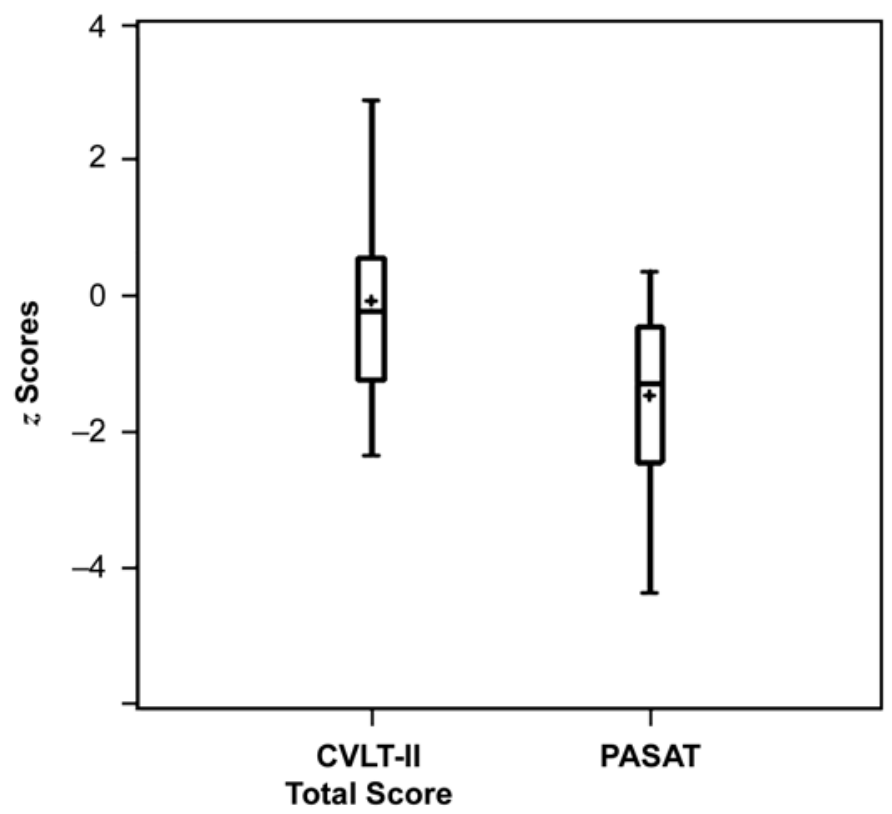

Figure 2.

A $z$ score distribution for Paced Auditory Serial Addition Test (PASAT) and California Verbal Learning Test, 2nd edition (CVLT-II) for all 49 subjects. Boxes represent 25th to 75th percentile, horizontal line is median, cross is mean, and vertical lines are maximum and minimum. 
Table 3.

Mean \pm standard deviation (SD) for neuropsychological tests and Perceived Deficits Questionnaire (PDQ) for subgroup subjects who received complete battery of tests $(n=38)$.

\begin{tabular}{lc}
\hline \multicolumn{1}{c}{ Variable } & Mean \pm SD \\
\hline PASAT & $35.7 \pm 12.1$ \\
CVLT-II Total Score & $42.0 \pm 12.7$ \\
CVLT-II Short Delay Free & $8.7 \pm 3.4$ \\
CVLT-II Short Delay Cued & $10.4 \pm 3.1$ \\
CVLT-II Long Delay Free & $8.5 \pm 3.9$ \\
CVLT-II Long Delay Cued & $9.8 \pm 3.6$ \\
Stroop & $33.3 \pm 14.0$ \\
SDMT & $44.8 \pm 11.0$ \\
COWAT & $35.6 \pm 11.0$ \\
UFOV & $9.7 \pm 14.7$ \\
BDI-IA & $7.4 \pm 5.3$ \\
PDQ & $55.4 \pm 13.2$ \\
BDI-IA $=$ Beck Depression Inventory-Amended; COWAT & $=$ \\
Controlled Oral Word Association Test; CVLT-II $=$ California Verbal \\
Learning Test, 2nd edition; PASAT = Paced Auditory Serial Addition \\
Test; SDMT = Symbol Digit Modalities Test; Stroop = Stroop color- \\
word test; UFOV = useful field of view. \\
\hline
\end{tabular}

Deleting the four subjects who were missing one item in the PDQ did not significantly alter the magnitude or the significance of the correlations between the PDQ and the BDI, PASAT, and CVLT-II total score.

\section{Correlations Between Complete Battery Tests and BDI-IA with PDQ of Subgroup Subjects}

Thirty-eight subjects had scores on the PASAT and CVLT-II total score worse than 0.5 SD below the mean and received the full neuropsychological battery. An analysis similar to the one performed on all the subjects did not show any significant correlations between the PDQ and any of the other neuropsychological tests. None of the additional tests in the battery correlated significantly with the BDI-IA. The correlation between the BDI-IA and PDQ persisted ( $r=0.55$; 95\% CI, 0.32 to $0.72 ; p=0.0004$ ). The correlation matrix is presented in Table 5.

\section{Correlations Between Individual Items in PDQ and PASAT, CVLT-II Total Score, and BDI-IA}

As an exploratory analysis, the correlations between the different specific items of the PDQ and the PASAT, CVLT-II total score, and BDI-IA were determined. Table 6 shows the correlations of the individual items of the PDQ with the CVLT-II total score and BDI-IA. Item 1, "Lose your train of thought when speaking?” $(r=0.51$; 95\% CI, 0.27 to $0.85 ; p=0.0002$ ), and item 3 , "Forgot what you

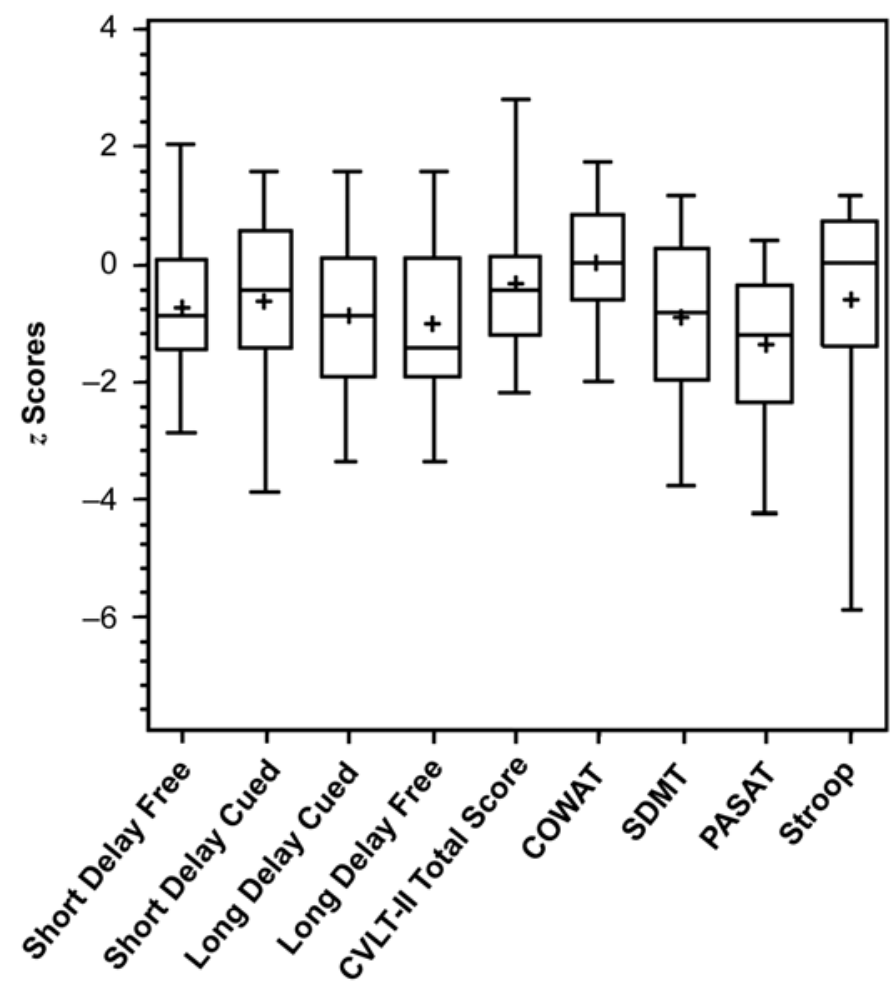

Figure 3.

A $z$ score distribution for all tests in neuropsychological battery on subgroup of 38 subjects. Boxes represent 25th to 75th percentile, horizontal line is median, cross is mean, and vertical lines are maximum and minimum. CVLT-II = California Verbal Learning Test, 2nd edition; COWAT = Controlled Oral Word Association Test; SDMT = Symbol Digit Modalities Test; PASAT = Paced Auditory Serial Addition Test; Stroop $=$ Stroop color-word test.

Table 4.

Correlation coefficients ( $r$ ) (95\% CIs) and $p$-values for Perceived Deficits Questionnaire (PDQ) and brief battery of tests on all subjects $(n=49){ }^{*}$

\begin{tabular}{|c|c|c|c|}
\hline Tests & CVLT Total & BDI-IA & PDQ \\
\hline PASAT & $\begin{array}{c}r=0.31 \\
(0.54 \text { to } 0.02) \\
p=0.03\end{array}$ & $\begin{array}{c}r=0.19 \\
(-0.10 \text { to } 0.45) \\
p=0.19\end{array}$ & $\begin{array}{c}r=0.18 \\
(-0.11 \text { to } 0.44) \\
p=0.20\end{array}$ \\
\hline CVLT-II Total & - & $\begin{array}{c}r=-0.27 \\
(-0.51 \text { to } 0.02) \\
p=0.06\end{array}$ & $\begin{array}{c}r=-0.17 \\
(-0.43 \text { to } 0.12) \\
p=0.25\end{array}$ \\
\hline BDI-IA & - & - & $\begin{array}{c}r=0.42 \\
(0.15 \text { to } 0.62) \\
p=0.003^{\dagger}\end{array}$ \\
\hline \multicolumn{4}{|c|}{$\begin{array}{l}{ }^{*} \text { Of this group, } 4 \text { subjects missed } 1 \text { of } 20 \text { questions in PDQ. Missing values } \\
\text { were replaced by mean response for whole group. } \\
{ }^{\dagger} \text { Statistically significant value. } \\
\text { BDI-IA = Beck Depression Inventory-Amended; CI = confidence interval; } \\
\text { CVLT-II = California Verbal Learning Test, 2nd edition; PASAT = Paced } \\
\text { Auditory Serial Addition Test. }\end{array}$} \\
\hline
\end{tabular}


Table 5.

Correlation coefficients $(r)$ and $p$-values for PDQ and complete neuropsychological battery tests for subgroup $(n=38)$.

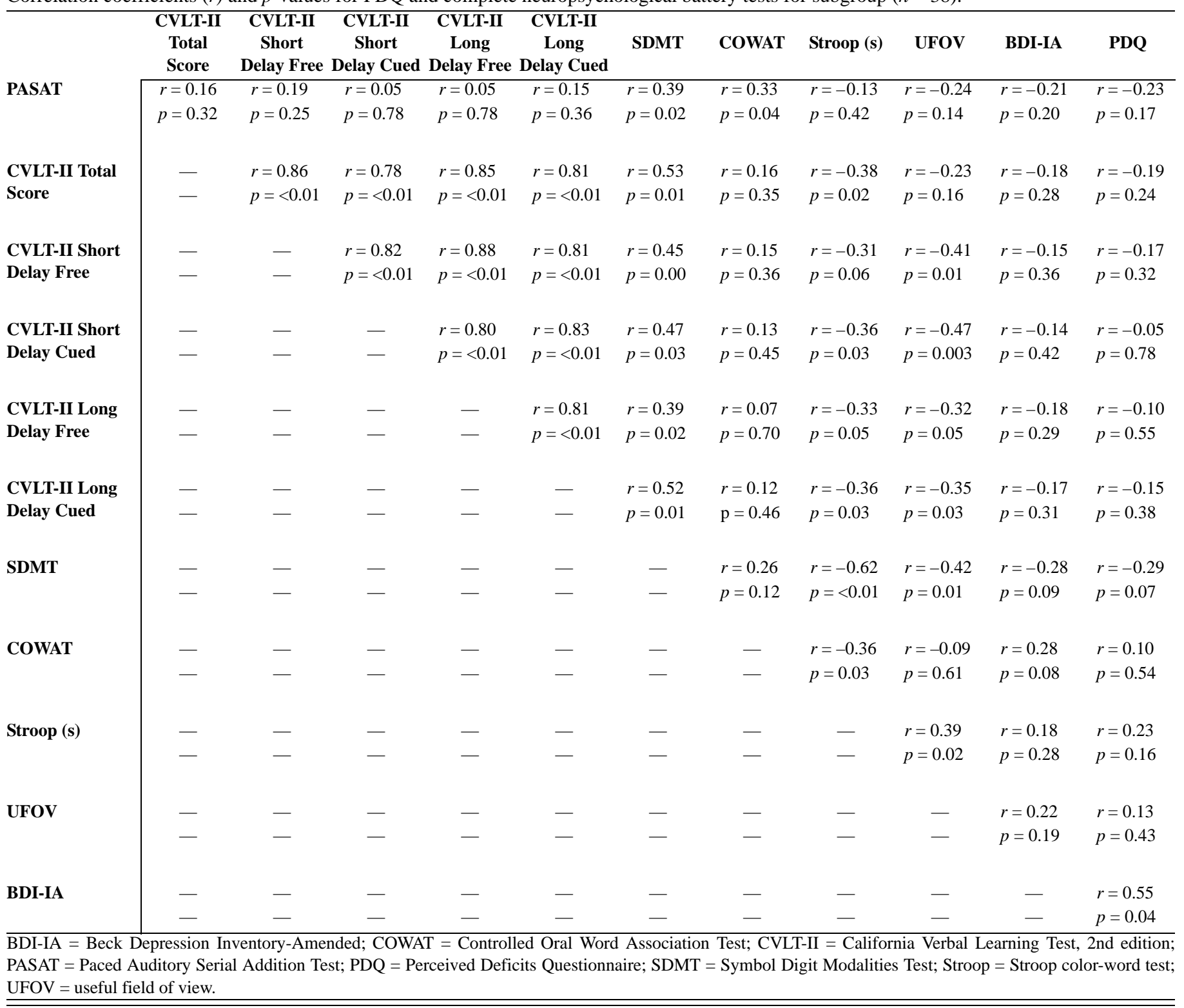

came into the room for?” ( $r=0.48$; $95 \%$ CI, 0.23 to 0.67 ; $p=0.0005)$, correlated highly with the BDI-IA. These correlations were significant after we adjusted for multiple comparisons. Item 14, "Forgot what you talked about after a telephone conversation?" and item 17, "Have trouble holding phone numbers in your head, even for a few seconds?" also showed a similar trend but did not reach the criteria for significance after we adjusted for multiple comparisons. None of the individual items had statistically significant correlations with the PASAT or the CVLT-II total score. However, item 2, "Have difficulty remembering the names of people, even the ones you have met several times?" ( $r=-0.31$; 95\% CI, -0.03 to $-0.54 ; p=0.03$ ), item 15 , "Forget to do things like turn off the stove or turn on your alarm clock?” $(r=-0.32 ; 95 \% \mathrm{CI},-0.04$ to $-0.55 ; p=$ 0.03 ), and item 16, "Feel like your mind went totally blank?” ( $r=-0.33$; 95\% CI, -0.05 to -0.56 ; $p=0.2$ ), showed modest correlations with the PASAT and not the BDI-IA, but these correlations did not reach the significance level after we adjusted for multiple comparisons. Item 18, "Forget what you did last weekend?" also showed a similar trend for the CVLT-II total score $(r=-0.30$; 
Table 6.

Correlation coefficients $(r)$ and $p$-values between individual items of PDQ and PASAT, CVLT-II total score, and BDI-IA for all subjects $(n=49)$.

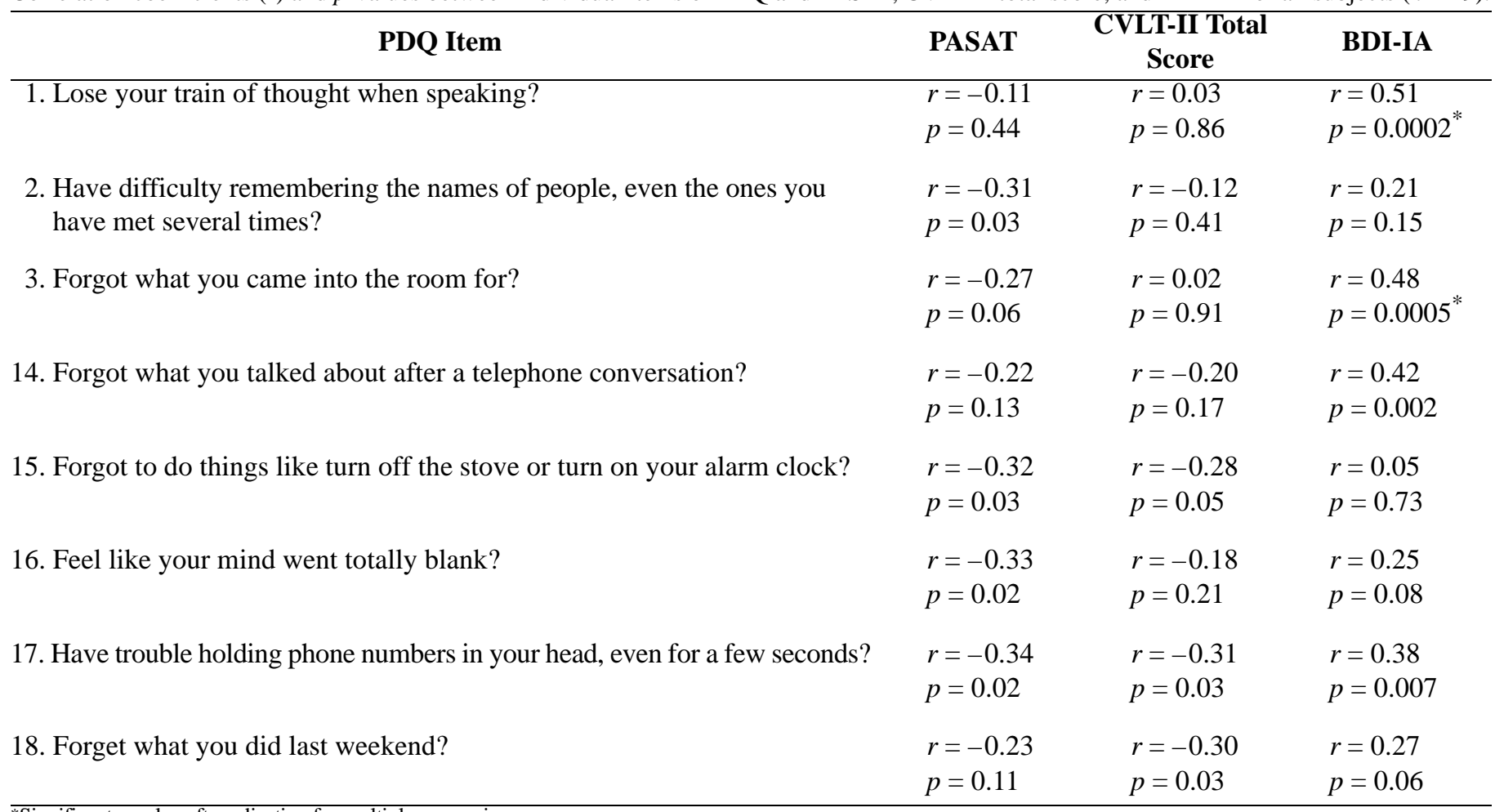

*Significant $p$-value after adjusting for multiple comparisons.

BDI-IA = Beck Depression Inventory-Amended; CVLT-II = California Verbal Learning Test, 2nd edition; PASAT = Paced Auditory Serial Addition Test; PDQ = Perceived Deficits Questionnaire.

$95 \% \mathrm{CI},-0.02$ to $-0.54 ; p=0.03)$. None of the other items on the PDQ showed significant correlations with the PASAT, CVLT-II, or BDI.

\section{DISCUSSION}

This study suggests that the self-reported cognitive difficulties measured by the PDQ relate more to depression than to objective cognitive impairment, even in this sample that excluded subjects with moderate or severe depression. The PDQ did not significantly correlate with two objective neuropsychological tests that measure verbal memory (CVLT-II) and sustained attention (PASAT), two domains that are frequently impaired in MS. The results of the analysis of the subjects who received the extended battery further conclude that the PDQ does not correlate with objective measures of attention, information processing, or phonemic fluency. These results agree with previous studies in people with MS where no correlation was found between self-reported impairment mea- sured with self-report instruments other than the PDQ and objective impairment in neuropsychological tests [15-17]. A study in people with MS did show significant correlations between self-report of memory on the Memory Rating Scale and delayed recall and attention tests, but this self-report instrument is oriented only to memory deficits [18]. Some self-report instruments may be better than others in correlating with objective measures of cognitive impairment. Studies in people with epilepsy [19], schizophrenia [20], and normal aging [21] have also failed to show a significant correlation between selfreport measures of cognitive impairment and objective testing. However, in longitudinal studies in aging and in human immunodeficiency virus, subjective cognitive deficits in initial testing were associated with developing cognitive difficulties on follow-up [22-23].

Some of the perceived deficits reported in the PDQ could possibly correlate with objective impairment in other domains that we did not test, such as executive function, visuospatial reasoning, or nonverbal memory. The tests we used measure the domains that are most 
frequently impaired in MS, so they would be expected to capture most of the cognitive impairment in the subjects. The content of most of the items in the PDQ suggests that they measure self-perceived deficits in working memory, verbal memory, and attention, so at least some of the items would be expected to correlate with the objective tests we used. This was not the case in the analysis of the individual items. Also possible is that the tests we used are not sensitive enough to capture slight impairment that subjects may still perceive. Further studies are needed to refine the PDQ as a measure of the impact of cognitive impairment on the QOL of MS patients by clarifying if some portions of it correlate with other objective tests not used in this study.

This study has other limitations. The sample size was small and the CIs do not exclude moderate correlation values, so moderate correlations were possibly missed due to chance and low power. Our study was also subject to selection bias because the subjects, the physician, or the family likely noted significant deficits that motivated the subjects to participate in the clinical trial; thus, it is possible that in a random sample of people with MS that includes subjects who are not symptomatic, the correlations between the PDQ and objective deficits would be higher.

We found that the PDQ had a moderately strong correlation with the BDI-IA. Similar results have been observed in other studies in MS using different selfreport instruments $[15,24]$. Researchers should examine the correlation of the BDI-IA with the PDQ more closely in future studies to determine if it is driven by the two items in the BDI-IA that relate to difficulties in making decisions and working. Some of the items in the PDQ correlated very highly with depression, and since other scales in the MSQLI already address mood and affect, these items may not be needed. Larger studies could help further clarify which items could be deleted because of their high correlation with depression and which items correlate best with objective cognitive deficits.

The MS Neuropsychological Screening Questionnaire, a scale that is administered to a caregiver or close family member, correlated with objective performance, while a similar scale given to the subjects did not [15]. This finding suggests that observed and self-perceived cognitive impairment may be two different domains with the degree of impairment observed by an independent informant more closely related to what is measured by cognitive tests. Additional scales given to an informant may be necessary to capture the impact of cognitive impairment on QOL.
Since the PDQ did not correlate with any of the objective cognitive tests used in this study, what the PDQ actually assesses is uncertain. The BDI-IA score only accounted for a small portion of the variance in the PDQ scores. Obtaining norms from healthy populations and populations with other diseases could help clarify if the variance in the PDQ not accounted for by the BDI-IA is normal variability or a dimension of impairment specific for MS that is not captured by objective tests.

\section{CONCLUSIONS}

This study has two important clinical implications. First, the results from this study emphasize the importance of addressing depression in people with MS who have cognitive complaints. Even in individuals with low levels of depression, like the subjects in this study, depression had a significant association with self-perceived impairment, while objective results did not. Second, the results also emphasize that objective neuropsychological testing helps clarify the domains that are impaired and the severity of impairment in people with MS who have cognitive complaints. Clarifying the domains and the severity of impairment will help the clinician diagnose and provide the necessary treatment and counseling. Careful evaluation of cognitive impairment will become even more important in the future as therapies for cognitive impairment in MS become available.

\section{ACKNOWLEDGMENTS}

Dr. Jesus Lovera is a Department of Veterans Affairs (VA) Special Fellow in MS and Dr. Bridget Bagert was a VA Research Enhancement Award Program Associate Investigator.

This material was based on work supported by the VA; the National Institutes of Health, grant P50 AT0006601; the National MS Society, grant PP0921; and The Nancy Davis Center Without Walls.

\section{REFERENCES}

1. Rao SM, Leo GJ, Bernardin L, Unverzagt F. Cognitive dysfunction in multiple sclerosis. I. Frequency, patterns, and prediction. Neurology. 1991;41(5):685-91. [PMID: 2027484 
2. Rao SM. Neuropsychology of multiple sclerosis. Curr Opin Neurol. 1995;8(3):216-20. [PMID: 7551121]

3. Rao SM, Leo GJ, Ellington L, Nauertz T, Bernardin L, Unverzagt F. Cognitive dysfunction in multiple sclerosis. II. Impact on employment and social functioning. Neurology. 1991;41(5):692-96. [PMID: 1823781]

4. Fischer JS, LaRocca NG, Miller DM, Ritvo PG, Andrews H, Paty DW. Recent developments in the assessment of quality of life in multiple sclerosis. Mult Scler. 1999;5(4):251-59. [PMID: 10467384]

5. Schultheis MT, Garay E, DeLuca J. The influence of cognitive impairment on driving performance in multiple sclerosis. Neurology. 2001;56(8):1089-94. [PMID: 11320184]

6. Shawaryn MA, Schultheis MT, Garay E, DeLuca J. Assessing functional status: exploring the relationship between the multiple sclerosis functional composite and driving. Arch Phys Med Rehabil. 2002;83(8):1123-29. [PMID: 12161835]

7. McDonald WI, Compston A, Edan G, Goodkin D, Hartung HP, Lublin FD, McFarland HF, Paty DW, Polman CH, Reingold SC, Sandberg-Wollheim M, Sibley W, Thompson A, Van den Noort S, Weinshenker BY, Wolinsky JS. Recommended diagnostic criteria for multiple sclerosis: guidelines from the international panel on the diagnosis of multiple sclerosis. Ann Neurol. 2001;50(1):121-27. [PMID: 11456302]

8. Beck AT, Ward CH, Mendelson M, Mock J, Erbaugh J. An inventory for measuring depression. Arch Gen Psychiatry. 1961;4:561-71. [PMID: 13688369]

9. Delis DC, Kramer JH, Kaplan E, Ober BA. CVLT-II: California Verbal Learning Test. 2nd ed. New York (NY): The Psychological Corporation; 1999. p. 172-203.

10. Lezak MD, Howieson DB, Loring DW, Hannay HJ, Fischer JS. Neuropsychological assessment. 4th ed. Oxford (England): Oxford University Press; 2004. p. 519-20.

11. Boringa JB, Lazeron RH, Reuling IE, Ader HJ, Pfennings L, Lindeboom J, De Sonneville LM, Kalkers NF, Polman CH. The brief repeatable battery of neuropsychological tests: normative values allow application in multiple sclerosis clinical practice. Mult Scler. 2001;7(4):263-67. [PMID: 11548987]

12. Ball KK, Roenker DL. UFOV: Useful field of view. New York (NY): The Psychological Corporation; 1999.

13. Spreen O, Strauss E. A compendium of neuropsychological tests. 1st ed. New York (NY): Oxford University Press; 1991. p. 52-53.
14. Rorsner B. Fundamentals of biostatistics. 5th ed. Florence (KY): Duxbury; 2000. p. 461.

15. Benedict RH, Munschauer F, Linn R, Miller C, Murphy E, Foley F, Jacobs L. Screening for multiple sclerosis cognitive impairment using a self-administered 15-item questionnaire. Mult Scler. 2003;9(1):95-101. [PMID: 12617275]

16. Taylor R. Relationships between cognitive test performance and everyday cognitive difficulties in multiple sclerosis. Br J Clin Psychol. 1990;29(Pt 2):251-53. [PMID: 2364208]

17. Beatty WW, Monson N. Metamemory in multiple sclerosis. J Clin Exp Neuropsychol. 1991;13(2):309-27. [PMID: 1688342]

18. Randolph JJ, Arnett PA, Higginson CI. Metamemory and tested cognitive functioning in multiple sclerosis. Clin Neuropsychol. 2001;15(3):357-68. [PMID: 11778774]

19. Elixhauser A, Leidy NK, Meador K, Means E, Willian MK. The relationship between memory performance, perceived cognitive function, and mood in patients with epilepsy. Epilepsy Res. 1999;37(1):13-24. [PMID: 10515171]

20. Prouteau A, Verdoux H, Briand C, Lesage A, Lalonde P, Nicole L, Reinharz D, Stip E. Self-assessed cognitive dysfunction and objective performance in outpatients with schizophrenia participating in a rehabilitation program. Schizophr Res. 2004;69(1):85-91. [PMID: 15145474]

21. Jungwirth S, Fischer P, Weissgram S, Kirchmeyr W, Bauer P, Tragl KH. Subjective memory complaints and objective memory impairment in the Vienna-Transdanube aging community. J Am Geriatr Soc. 2004;52(2):263-68. [PMID: 14728638]

22. Beason-Hazen S, Nasrallah HA, Bornstein RA. Self-report of symptoms and neuropsychological performance in asymptomatic HIV-positive individuals. J Neuropsychiatry Clin Neurosci. 1994;6(1):43-49. [PMID: 8148636]

23. Cipolli C, Neri M, Andermarcher E, Pinelli M, Lalla M. Selfrating and objective memory testing of normal and depressed elderly. Aging (Milano). 1990;2(1):39-48. [PMID: 2094354]

24. Randolph JJ, Arnett PA, Freske P. Metamemory in multiple sclerosis: exploring affective and executive contributors. Arch Clin Neuropsychol. 2004;19(2):259-79. [PMID: 15010090]

Submitted for publication September 7, 2004. Accepted in revised form January 19, 2005. 